\title{
Discovery of Centrosomal Protein 70 as an Important Player in the Development and Progression of Breast Cancer
}

Xingjuan Shi, ${ }^{\star \dagger}$ Dengwen $\mathrm{Li}^{\dagger}{ }^{\dagger}$ Yujue Wang, ${ }^{*}$ Shiyu Liu, ${ }^{\dagger}$ Juan Qin, ${ }^{\dagger}$ Jun Wang, ${ }^{\dagger}$ Jie Ran, ${ }^{\star}$ Yu Zhang, ${ }^{*}$ Qinghai Huang, ${ }^{*}$ Xiangdong Liu, ${ }^{*}$ Jun Zhou, ${ }^{\dagger \ddagger}$ and Min Liu ${ }^{\ddagger}$

From the Key Laboratory of Developmental Genes and Human Disease, * Institute of Life Sciences, Southeast University, Nanjing; the State Key Laboratory of Medicinal Chemical Biology, ${ }^{\dagger}$ College of Life Sciences, Nankai University, Tianjin; and the Institute of Biomedical Sciences, ${ }^{\ddagger}$ College of Life Sciences, Shandong Provincial Key Laboratory of Animal Resistance Biology, Shandong Normal University, Jinan, China

\author{
Accepted for publication \\ November 8, 2016. \\ Address correspondence to \\ Xingjuan Shi, Ph.D., Key Labora- \\ tory of Developmental Genes and \\ Human Disease, Institute of Life \\ Sciences, Southeast University, \\ Nanjing 210096, China; or Min \\ Liu, Ph.D., Institute of Biomedical \\ Sciences, College of Life Sciences, \\ Shandong Provincial Key Labora- \\ tory of Animal Resistance Biology, \\ Shandong Normal University, \\ Jinan 250014, China. E-mail: \\ xingjuanshi@seu.edu.cn or \\ minliu@sdnu.edu.cn.
}

\begin{abstract}
Centrosome abnormalities have been implicated in the development and progression of breast cancer. However, the molecular players involved in the above processes remain largely uncharacterized. Herein, we identify centrosomal protein 70 (Cep70) as an important factor that mediates breast cancer growth and metastasis. Cep70 is up-regulated in breast cancer tissues and cell lines, and its expression is closely correlated with several clinicopathologic variables associated with breast cancer progression. Mechanistic studies reveal that the up-regulation of Cep70 in breast cancer occurs at the mRNA level and is independent of gene amplification. Cep70 promotes breast cancer cell proliferation and colony formation in vitro and increases tumor growth in mice. In addition, Cep70 stimulates breast cancer cell migration and invasion in vitro. Bioluminescence imaging analysis further shows that Cep70 enhances breast cancer lung metastasis in mice. Together, these results demonstrate a critical role for Cep70 in the development and progression of breast cancer and have important implications in the diagnosis and therapy of this malignancy. (Am J Pathol 2017, 187: 679-688; http://dx.doi.org/10.1016/ j.ajpath.2016.11.005)
\end{abstract}

Breast cancer is one of the leading causes of cancer deaths in women. ${ }^{1}$ The cause of breast cancer is complex and has been reported to have both genetic and environmental contributions. Treatment of breast cancer frequently involves surgical resection in combination with systemic treatments that can include cytotoxic, hormonal, and/or immunotherapeutic agents. Early breast cancer is frequently effectively managed with surgery combined with radiotherapy or chemotherapy. However, mortality rates remains high for locally advanced and metastatic cancer. ${ }^{2}$ Over the past decades, the use of well-established biomarkers has improved outcomes for breast cancer by facilitating targeted selection of therapeutics most likely to provide benefit. Markers such as estrogen receptor, progesterone receptor, and human epidermal growth factor receptor 2 have substantial prognostic value and play important roles in selecting patients for endocrine or trastuzumab therapies. ${ }^{3}$ To continue to develop more reliable biomarkers and therapeutics, it is important to identify additional molecular players that drive the development and progression of this malignancy.

Supported by the National Basic Research Program of China grant 2012CB945002 (J.Z.); the National Natural Science Foundation of China grants 31301113 (X.S.), 31130015 (J.Z.), and 81270295 (X.L.); the Tianjin Natural Science Foundation grant 15JCYBJC49300 (J.Z.); the Natural Science Foundation of Jiangsu Province grants BK20130607 (X.S.) and SBK2015020697 (X.L.); Jiangsu Provincial Innovation Fund (X.L.), and the State Key Laboratory of Medicinal Chemical Biology (X.S.). X.S. is an assistant fellow and X.L. is a fellow at the Collaborative Innovation Center for Cardiovascular Disease Translational Medicine of Jiangsu Province, China.

X.S. and D.L. contributed equally to this work.

Disclosures: None declared. 
The centrosome plays a central role in organizing the microtubule network and in coordinating bipolar spindle formation during mitosis. ${ }^{4}$ Centrosome abnormalities, such as supernumerary centrioles, abnormal centrosome number or volume, altered phosphorylation of centrosomal proteins, and modification and accumulation of pericentriolar materials, are common in human cancers. ${ }^{5,6}$ In general, centrosome aberrations are positively associated with advanced cancer grade and metastasis. ${ }^{7}$ Thus, mutations or changes in the expression of proteins that regulate the centrosome and its activities may contribute to breast cancer development and progression. Centrosomal protein 70 (Cep70) localizes to the centrosome throughout the cell cycle via an interaction with $\gamma$-tubulin, ${ }^{8}$ and it has been shown to participate in a variety of cellular processes such as microtubule organization, cell division, cell migration, and ciliogenesis. ${ }^{8-14}$ However, a pathologic role for Cep70 in breast cancer has not been explored. In this study, we present the first evidence to our knowledge that Cep70 plays an important role in breast cancer growth and metastasis.

\section{Materials and Methods}

\section{Ethics Statement}

Investigation has been conducted in accordance with the ethical standards according to the Declaration of Helsinki and the national and international guidelines, and has been approved by the authors' institutional review board.

\section{Materials}

Sulforhodamine B (SRB), MTT, and DAPI, and antibodies against $\beta$-actin were purchased from Sigma-Aldrich (St. Louis, MO). The anti-Cep70 antibody was generated as described previously. ${ }^{8}$ Antibodies against Ki-67 and horseradish peroxidase-conjugated secondary antibodies were obtained from Santa Cruz Biotechnology (Santa Cruz, CA). Fluorometric TUNEL (terminal deoxynucleotidyl transferase-mediated dUTP nick-end labeling) System was purchased from Promega (Madison, WI). Human Cep70 siRNAs (siCep70 no. 1: 5'-GAGGAUGAAUCACUAAGUA-3'; siCep70 no. 2: 5'-CCUCAUACCGUCUUGGAUA$3^{\prime}$ ), mouse Cep70 siRNAs (simCep70 no. 1: 5'-GCAACUCAAGGAAUCCAAA-3'; simCep70 no. 2: 5'-GUGUAAACUGCAUUCGUAA- ${ }^{\prime}$ ), and luciferase control siRNA (5'-CGUACGCGGAAUACUUCGA-3') were synthesized by RiboBio (Guangzhou, China).

\section{Cell Culture and Transfection}

184A1 normal human breast epithelial cells and all human breast cancer cells were purchased from the ATCC (Manassas, VA). 184A1 cells were cultured in Dulbecco's modified Eagle's medium (DMEM)/F12. MCF7, BT474, and HS578T cells were cultured in the Dulbecco's modified Eagle's medium. T47D, MDA-MB-231, and BT549 cells were cultured in RPMI 1640 medium. The 4T1-luc luciferaseexpressing mouse breast cancer cells were cultured in the RPMI 1640 medium. All cell lines were maintained at $37^{\circ} \mathrm{C}$ in a 5\% $\mathrm{CO}_{2}$ humidified atmosphere. siRNAs were transfected with the lipofectamine reagent (Invitrogen, Carlsbad, CA) according to the manufacturer's manual.

\section{Immunohistochemistry}

Human breast cancer tissue samples and adjacent tissue samples were obtained from patients who underwent surgical resection at Shanxian Dongda Hospital. The tissue sections were deparaffinized and rehydrated with xylene and graded alcohols. After antigen retrieval and inactivation of endogenous peroxidase, sections were blocked with goat serum and incubated with primary antibodies. The sections were then incubated with biotinylated secondary antibody and streptavidin-biotin-peroxidase, using diaminobenzidine as the chromogen substrate, and finally hematoxylin counterstaining was performed. The expression of Cep70 was graded according to the staining intensity $(0=$ negative; $1=$ low; $2=$ medium; $3=$ high) and the percentage of stained cells $(0=0 \%$ stained; $1=1 \%$ to $25 \%$ stained; $2=26 \%$ to $50 \%$ stained; $3=51 \%$ to $100 \%$ stained) as described previously. ${ }^{15,16}$

\section{Quantitative Real-Time RT-PCR}

Total RNA was prepared using the Trizol reagent (Invitrogen), and cDNA was then synthesized from the RNA using the Superscript kit (Invitrogen). Genomic DNA was isolated using a tissue DNA isolation kit (Qiagen, Valencia, CA). Quantitative real-time PCR was performed using the SYBR Premix Ex Taq reagent (Takara, Kyoto, Japan) as described previously. ${ }^{17,18}$ Experiments were performed in triplicate and repeated at least three times.

\section{Fluorescence in Situ Hybridization Analysis}

The Cep70-specific probe was labeled with fluoresceindUTP (green) using the Bioprime DNA labeling system (Invitrogen) and hybridized with cellular nuclei. Nuclei were then stained with DAPI and examined with a Zeiss Axio Observer A1 fluorescence microscope (Carl Zeiss $\mathrm{GmbH}$, Jena, Germany).

\section{Immunoblotting}

Cells were lysed in a buffer containing $1 \%$ Triton X-100,

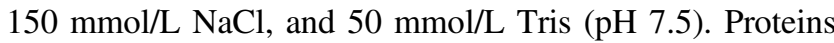
were resolved by SDS-PAGE and transferred onto polyvinylidene difluoride membranes (Millipore, Billerica, MA). The membranes were blocked in Tris-buffered saline containing $0.2 \%$ Tween 20 and 5\% fat-free dry milk and incubated with primary antibodies and then horseradish 
peroxidase-conjugated secondary antibodies as described previously. ${ }^{19,20}$ Specific proteins were visualized with enhanced chemiluminescence detection reagent according to the manufacturer's instructions (Pierce, Rockford, IL).

\section{In Vitro Cell Proliferation Assays}

T47D and MCF7 cells transfected with siRNAs were seeded in 96-well plates. For SRB assays, cells were fixed with 50\% trichloroacetic acid, stained with $0.4 \%$ SRB, and washed with $1 \%$ acetic acid. The protein-bound dye was then extracted with $10 \mathrm{mmol} / \mathrm{L}$ Tris base to determine the absorbance at $562 \mathrm{~nm}$. MTT assays were performed by using the Vybrant MTT cell proliferation assay kit (Thermo Fisher Scientific, Waltham, MA) according to the manufacturer's instructions. For each experiment, data are given from three independent experiments performed in technical triplicates.

\section{Colony Formation Assays}

Cells were seeded in 6-well plates and incubated for 2 weeks. The colonies were then fixed with methanol and stained with $0.1 \%$ crystal violet. Photographs were taken, and the number of colonies at each well was counted. For soft agar assays, cells were mixed with $0.3 \%$ agar before seeding.

\section{Cell Motility Assays}

Cell motility assays were performed as described previously. $^{21}$ For in vitro healing experiments, confluent
A

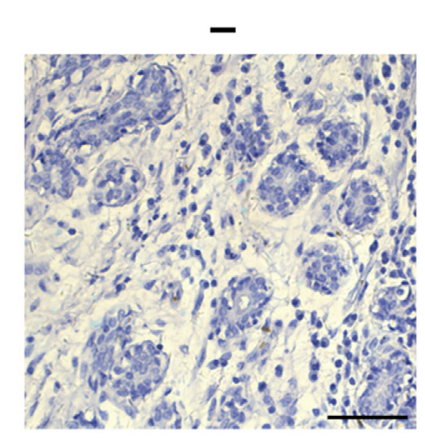

B

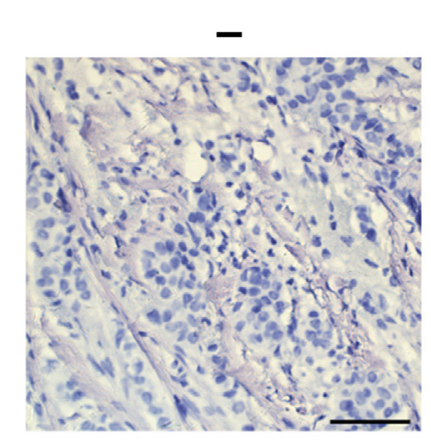

\section{Cancer tissue}
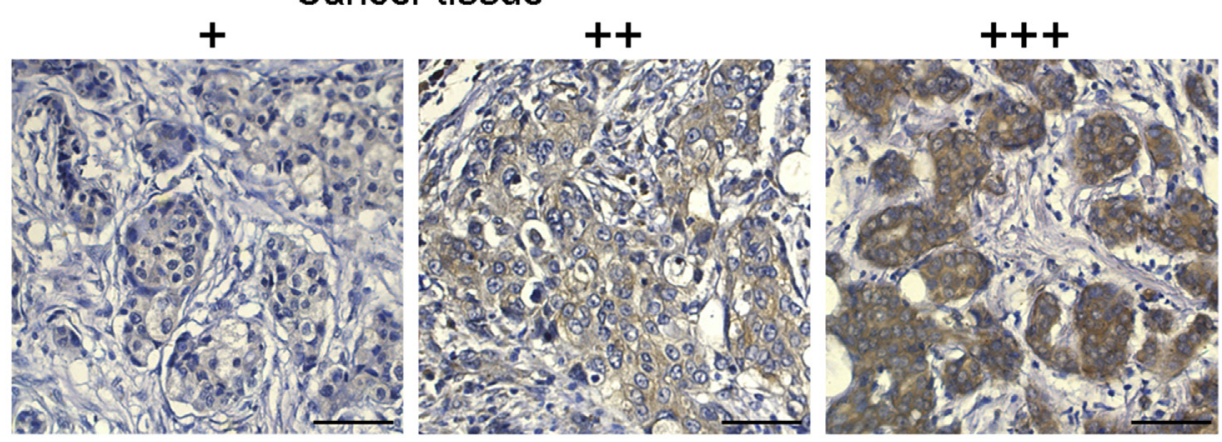
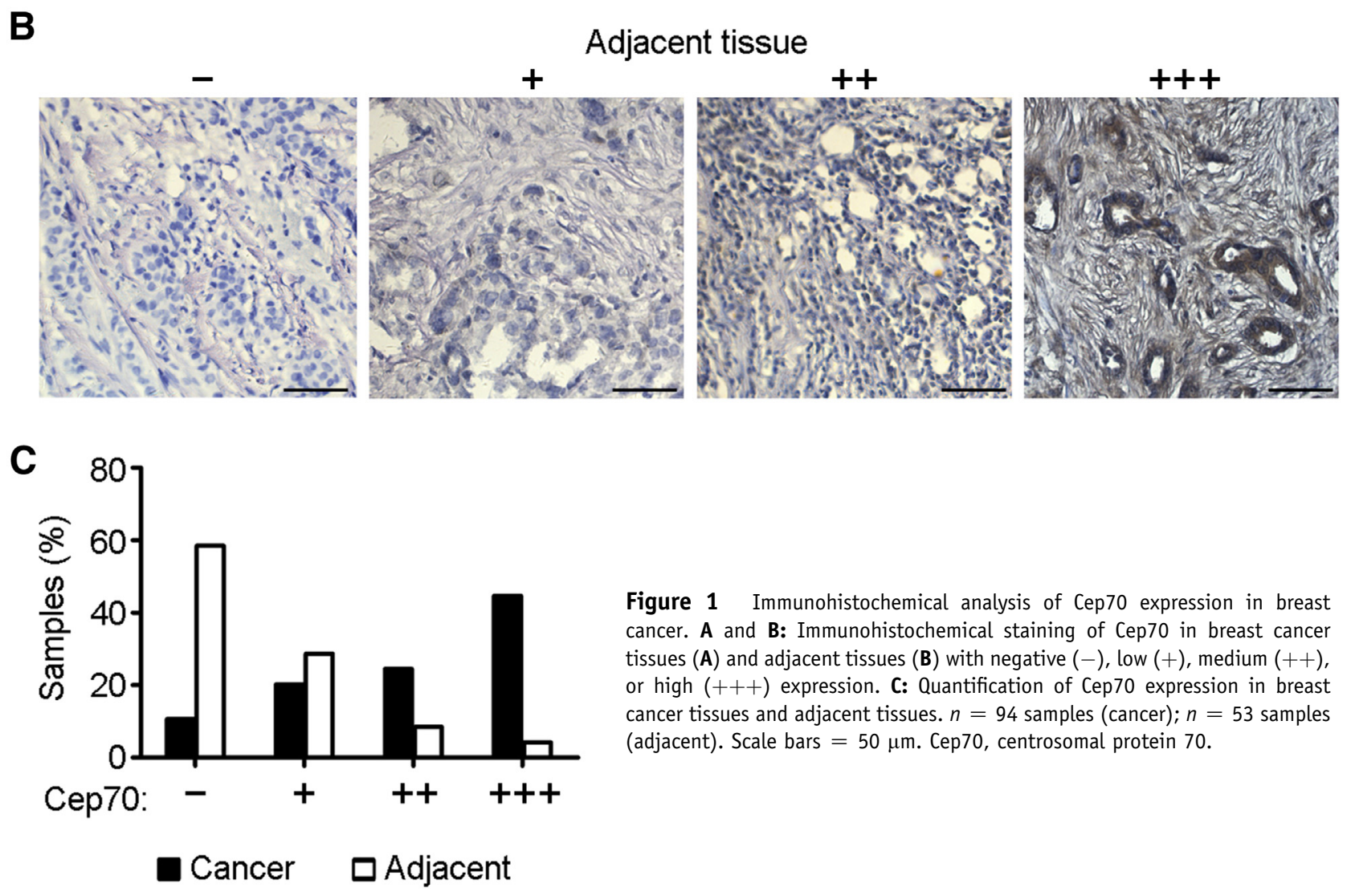

Figure 1 Immunohistochemical analysis of Cep70 expression in breast cancer. A and B: Immunohistochemical staining of Cep70 in breast cancer tissues (A) and adjacent tissues (B) with negative $(-)$, low $(+)$, medium $(++)$, or high $(+++)$ expression. C: Quantification of Cep70 expression in breast cancer tissues and adjacent tissues. $n=94$ samples (cancer); $n=53$ samples (adjacent). Scale bars $=50 \mu \mathrm{m}$. Cep70, centrosomal protein 70 . 
monolayers of cells cultured in 24-well plates in serum-free medium were scratched with a $10-\mu \mathrm{L}$ pipette tip to generate the wound. Cells were washed with phosphate-buffered saline, and complete medium was added to allow wound healing. Photographs of the wound were taken at 0 and 14 hours to determine the extent of wound closure. For Transwell migration assays, cells suspended in serum-free medium were added to the upper chamber of inserts (BD Falcon, New Jersey) precoated with Matrigel, and the chamber was placed in a 24-well plate containing complete medium. After 24 hours, cells on the inside of the inserts were removed with a cotton swab, and cells on the underside of the insert were fixed with $4 \%$ paraformaldehyde, stained with the crystal violet solution, and photographed. Each experiment was repeated at least three times. Assays were performed in triplicate wells, and the number of migrated or invaded cells in each well was counted in three randomly selected microscopic fields.

\section{Tumor Growth and Metastasis in Mice}

For tumor growth measurement, MCF7 cells were injected subcutaneously into the right flank of female athymic nude mice, and tumor volumes were measured every week. After the mice were sacrificed, tumors were isolated, photographed, weighed, and sectioned for immunohistochemistry staining. To analyze tumor metastasis, 4T1-luc cells were injected into the lateral tail vein of female $\mathrm{BALB} / \mathrm{c}$ mice as described previously. ${ }^{22}$ Luciferin $(150 \mathrm{mg} / \mathrm{kg}$ ) was injected intraperitoneally 10 minutes before imaging. Metastases were monitored with an IVIS bioluminescence imaging system (Xenogen, Alameda, CA). At the end point of experiments, mice were sacrificed, and tumors were harvested for bioluminescence imaging or sectioned for hematoxylin and eosin staining. Eight mice were used for each group in the animal studies.

\section{Statistical Analysis}

Analysis of statistical significance was performed by the $t$-test for comparison between two groups and by the analysis of variance test for multiple comparisons. Correlation coefficient was calculated by the Spearman's rank correlation test.

\section{Results}

Immunohistochemical Analysis of Cep70 Expression in Breast Cancer

We collected 94 breast cancer tissue samples and 53 adjacent tissue samples from patients who underwent surgical

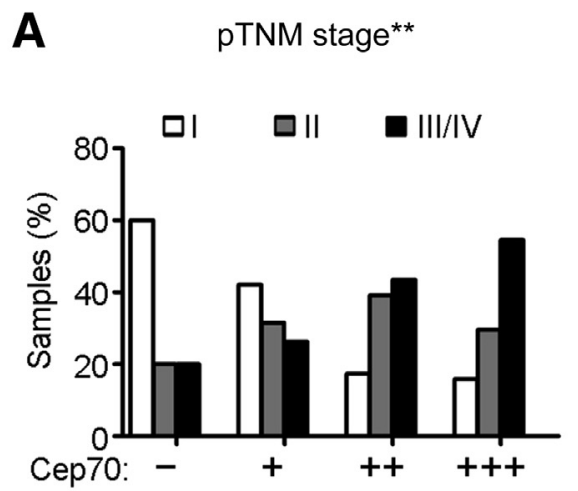

D

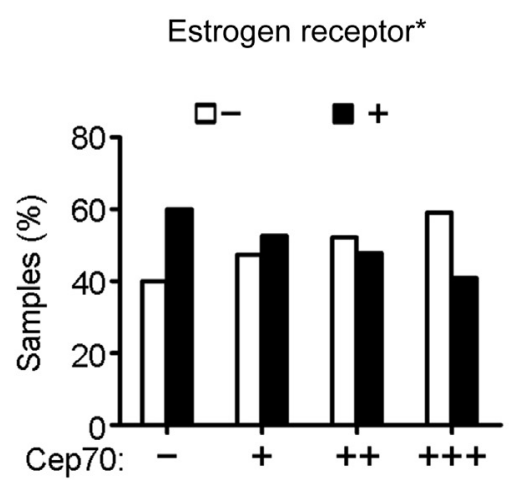

B

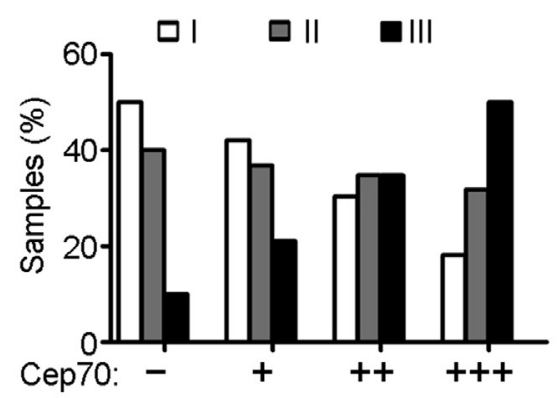

C Lymph node metastasis ${ }^{* \star}$

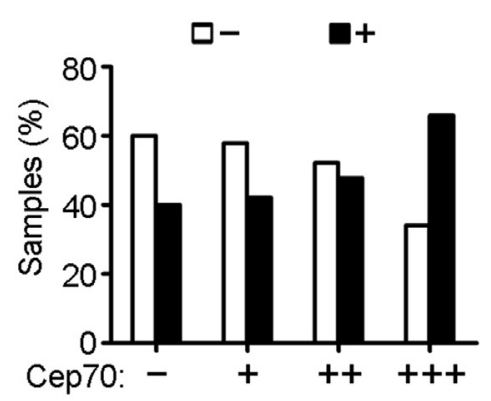

E Progesterone receptor*

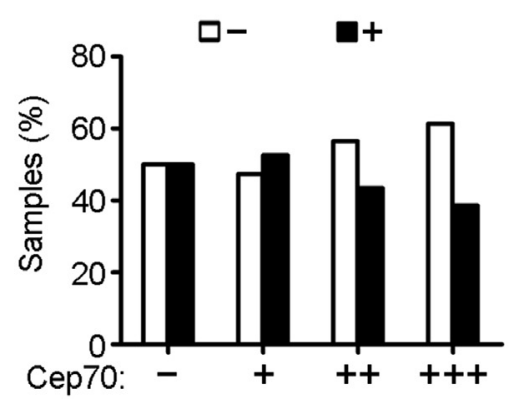

Figure 2 Examination of the correlation between Cep70 expression and clinicopathologic variables. Cep70 expression in breast cancer was examined by immunohistochemical staining as described in Figure 1, and its correlation with pTNM stage (A), histologic grade (B), lymph node metastasis (C), estrogen receptor status (D), and progesterone receptor status $(\mathbf{E})$ was determined by the Spearman's rank correlation test. ${ }^{*} P<0.05,{ }^{* *} P<0.01$. Cep70, centrosomal protein 70; pTNM, pathologic tumor node metastasis. 
A

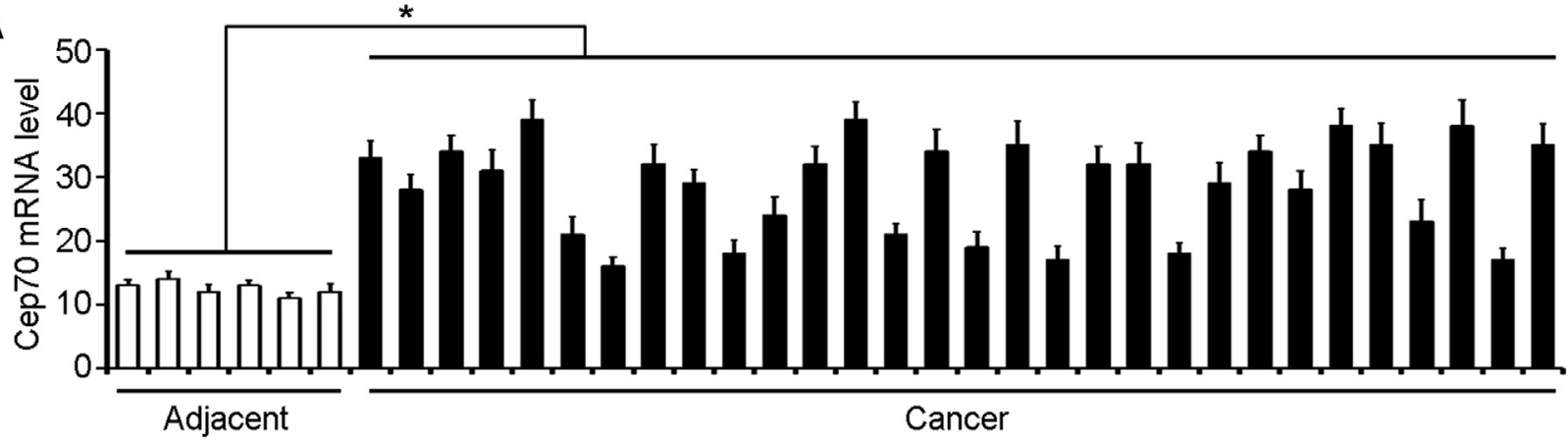

B

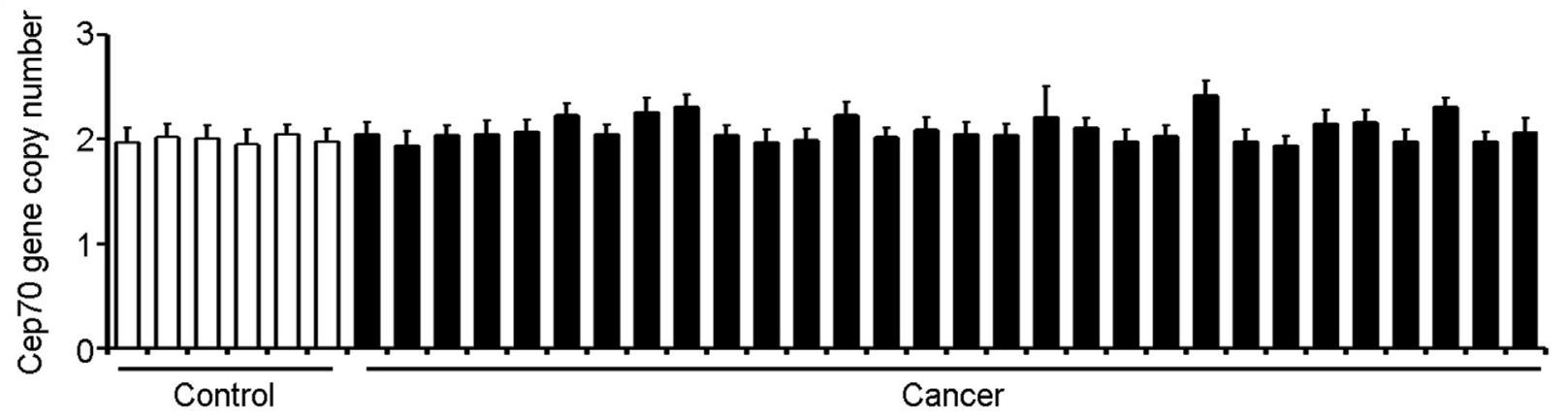

C

D

E

$\mathbf{F}$

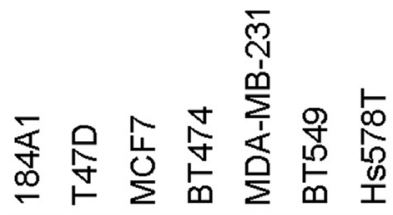

Cep70

Actin
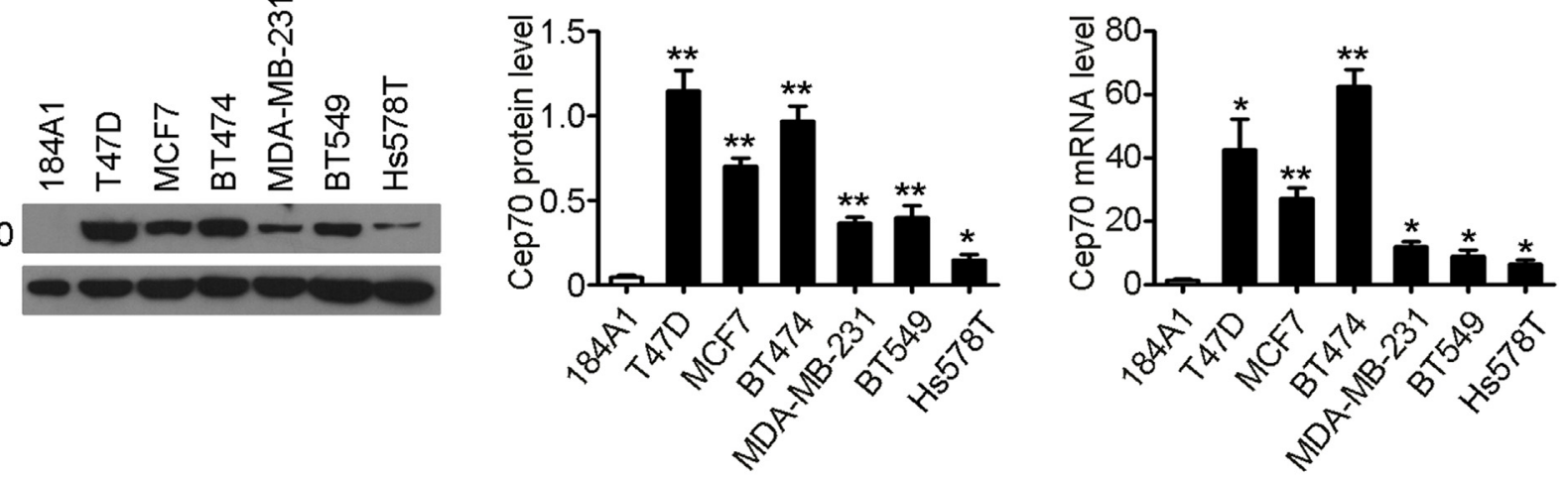

184A1

MCF7
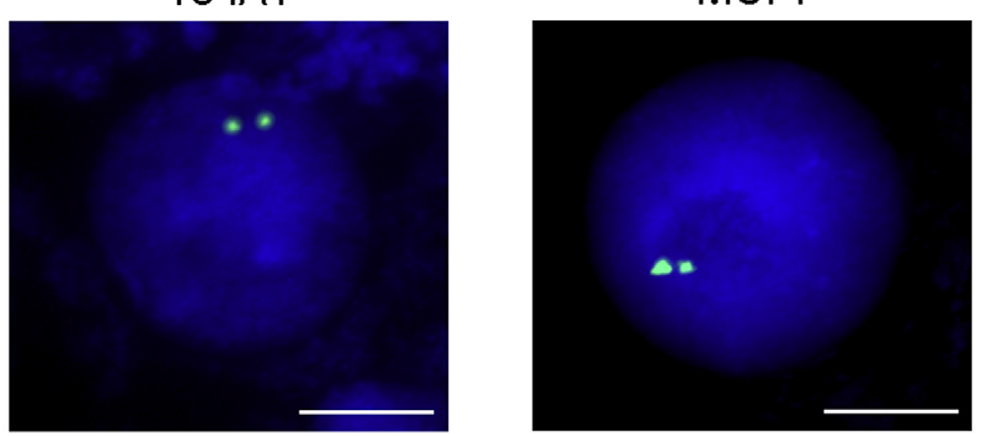

MDA-MB-231

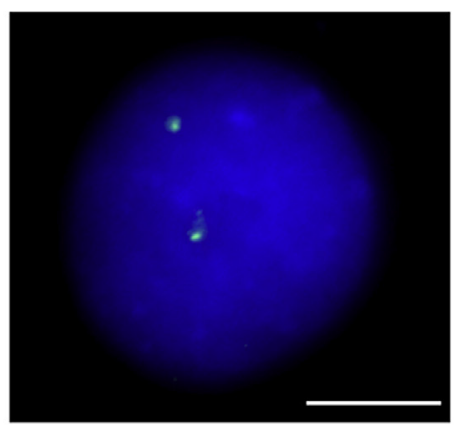

Figure 3 Analysis of Cep70 mRNA levels and gene copy numbers in breast cancer tissues and cell lines. A: Quantitative real-time RT-PCR analysis of Cep70 mRNA levels in breast cancer tissues and adjacent tissues. B: Quantitative real-time PCR analysis of Cep70 gene copy number in breast cancer. DNA isolated from blood leukocytes of healthy individuals served as control. C and D: Immunoblot analysis (C) and quantification (D) of Cep70 protein level in $184 A 1$ normal breast epithelial cells and breast cancer cell lines. E: Quantitative real-time RT-PCR analysis of Cep70 mRNA level. F: Examination of Cep70 gene copy number by FISH. Cep70-hybridizing loci are visible as green spots. Nuclei were stained with DAPI (blue). Data are expressed as means \pm SD. ${ }^{*} P<0.05$, ${ }^{*} P<0.01$ versus 184A1. Scale bars $=5 \mu \mathrm{m}$. Cep70, centrosomal protein 70; FISH, fluorescence in situ hybridization. 
resection as a part of their treatment plan. Immunohistochemical staining was performed to analyze Cep70 expression in these samples. The localization pattern of Cep70 was mainly in cytoplasm in breast cancer tissues, which were as similar as in adjacent tissues (Figure 1, A and B). The samples were subsequently categorized into four groups based on the intensity of Cep70 staining and the percentage of stained cells.

In comparison with adjacent tissues, expression of Cep70 in breast cancer tissues was remarkably increased. As shown in Figure 1C, approximately 59\% of adjacent tissues lacked Cep70 expression, whereas only $11 \%$ of breast cancer tissues exhibited absence of Cep70 expression. Among the samples positive for Cep70 expression, we found that $45 \%$ of breast cancer tissues showed high expression $(+++)$, but only $4 \%$ of adjacent tissues had high expression (Figure 1C). These results indicate that expression of Cep70 is significantly up-regulated in human breast cancer tissues.

\section{Examination of the Correlation between Cep70 Expression and Clinicopathologic Variables}

Given the elevated expression of Cep70 in breast cancer tissues, we examined the relation between Cep70 expression and clinical characteristics associated with breast cancer.
The chart outlining the features of breast cancer samples is shown in Supplemental Table S1. We analyzed several clinicopathologic variables for each of the 94 breast cancer tissue samples, including pathologic tumor node metastasis stage, histologic grade, lymph node metastasis, estrogen receptor status, and progesterone receptor status. We found that Cep70 expression was significantly positively correlated with higher pathologic tumor node metastasis stage (Figure 2A), higher histologic grade (Figure 2B), and higher incidence of lymph node metastasis (Figure 2C). In contrast, a negative correlation was observed between Cep70 and estrogen receptor expression (Figure 2D) and between Cep70 and progesterone receptor expression (Figure 2E). These data suggest that Cep70 may play an important role in the pathogenesis of hormone receptor-positive breast cancer.

\section{Analysis of Cep70 mRNA Levels and Gene Copy Numbers in Breast Cancer Tissues and Cell Lines}

We next performed quantitative real-time RT-PCR to examine the expression of Cep70 mRNA. In comparison with adjacent tissues, breast cancer tissue samples exhibited significantly increased Cep70 mRNA levels, with an average 2.3-fold increase (Figure 3A). Gene copy number gain is a mechanism that frequently leads to mRNA
A

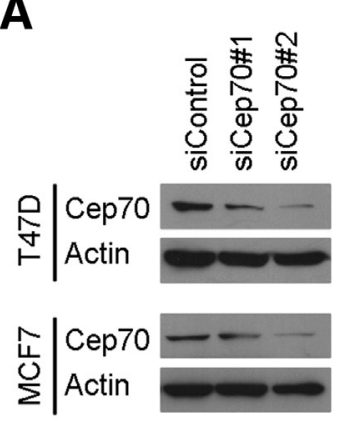

D

$$
\begin{aligned}
& \square \text { siControl } \\
& \square \text { siCep70\#1 } \\
& \square \text { siCep70\#2 }
\end{aligned}
$$

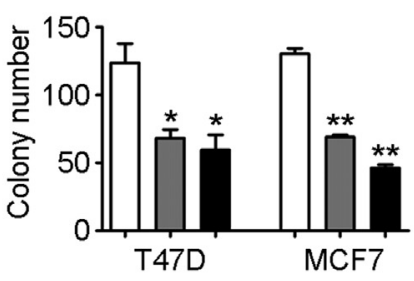

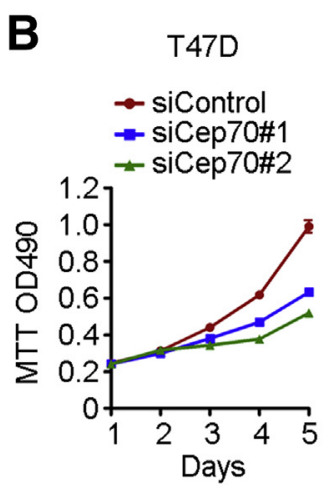

E
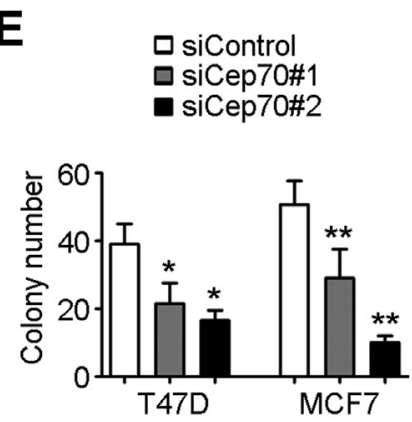
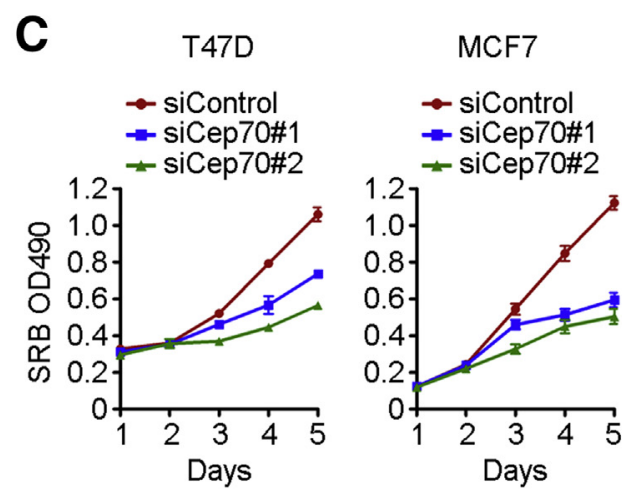

$\mathbf{F}$
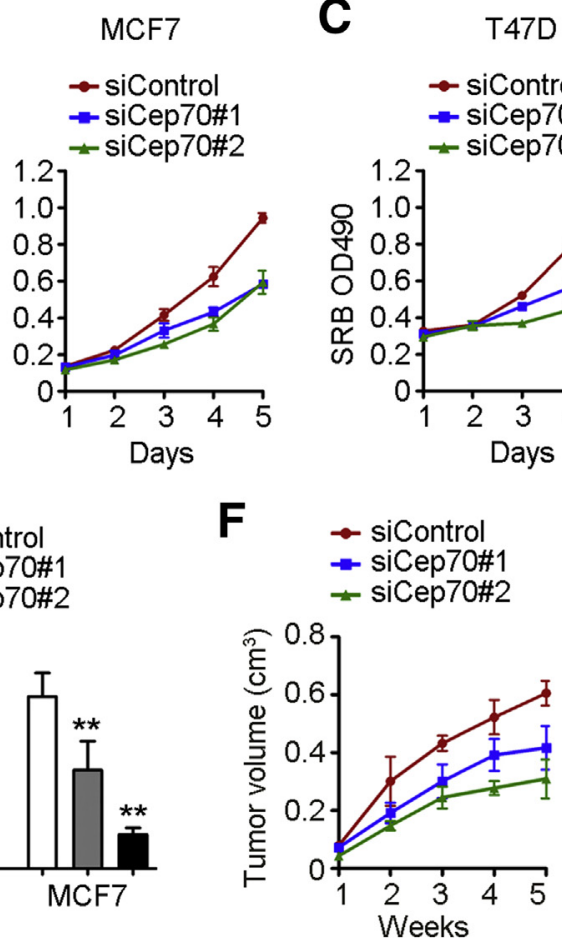

G
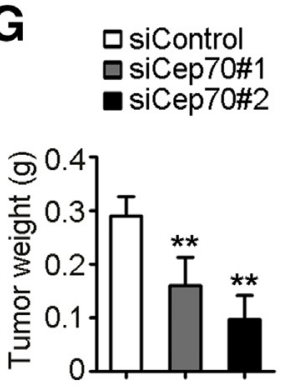

Figure 4 Effects of Cep70 on breast cancer cell proliferation and colony formation in vitro and tumor growth in vivo. A: Immunoblot analysis of Cep70 and $\beta$-actin expression in T47D and MCF7 cells transfected with control or Cep70 siRNAs. B and C: Analysis of proliferation of cells transfected with control or Cep70 siRNAs using MTT (B) and SRB (C) assays. D and E: Quantification of the number of colonies formed from control or Cep70 siRNA-transfected T47D and MCF7 cells by crystal violet staining (D) and soft agar (E) assays. F: MCF7 cells transfected with control or Cep70 siRNAs were injected subcutaneously into the right flanks of female athymic nude mice. Tumor volume was then measured each week. G: Experiments were performed as described in $\mathbf{F}$, and mice were sacrificed 5 weeks after injection. Tumors were then isolated and weighed. ${ }^{*} P<0.05,{ }^{*} P<0.01$ versus control. Data are expressed as means \pm SD. Cep70, centrosomal protein 70; si, small interfering; SRB, sulforhodamine B. 
overexpression in breast cancer. ${ }^{23}$ Thus, to investigate whether Cep70 gene copy number is altered in breast cancer tissues, we performed quantitative real-time PCR analysis of DNA isolated from breast cancer tissues, using DNA isolated from blood leukocytes of healthy individuals as control. We found that the Cep70 gene copy number in breast cancer tissues was similar to that of the control samples (Figure 3B). In addition, we analyzed data sets from The Cancer Genome Atlas database in the Oncomine platform. Cep70 mRNA was markedly increased in breast cancer tissues compared with normal breast tissues (Supplemental Figure S1A), whereas no significant difference was observed in Cep70 gene copy number between normal and breast cancer tissues (Supplemental Figure S1B).

To further validate the increased expression of Cep70 in breast cancer, we examined Cep70 expression in 184A1 normal breast epithelial cells and breast cancer cell lines by immunoblotting. Cep70 was almost undetectable in 184A1 cells, whereas its expression was significantly elevated in all of the breast cancer cell lines examined (Figure 3, C and D). In addition, quantitative real-time RT-PCR revealed that Cep70 mRNA levels were significantly increased in breast cancer cell lines (Figure 3E). To analyze whether Cep70 gene amplification was present in breast cancer cell lines, fluorescence in situ hybridization was performed to measure Cep70 gene copy numbers. Cep70 gene copy number did not differ between breast cancer cells and normal breast epithelial cells (Figure 3F). These data suggest that the overexpression of Cep70 in breast cancer is independent of gene copy number gain.

\section{Effects of Cep70 on Breast Cancer Cell Proliferation and Colony Formation in Vitro and Tumor Growth in Vivo}

To characterize the role of Cep70 in the pathogenesis of breast cancer, we explored its potential function in the proliferation of breast cancer cells. Cep70 expression was decreased in T47D and MCF7 breast cancer cells after transfection with specific siRNAs (Figure 4A). With the use of SRB and 3MTT assays, we found that knockdown of Cep70 expression significantly inhibited proliferation of breast cancer cells (Figure 4, B and C). To further assess the role of Cep70 in the proliferation of breast cancer cells, we quantified the number of colonies formed either on plastic or in soft agar by control or Cep70 siRNA-transfected cells. In comparison with cells transfected with control siRNAs, cells transfected with Cep70 siRNAs exhibited a significantly reduced number of colonies formed in both assays (Figure 4, D and E). These data indicate that Cep70 is important for both anchorage-dependent and -independent growth of breast cancer cells.

To explore the role of Cep70 in breast tumorigenesis in vivo, control or Cep70 siRNA-transfected MCF7 cells were injected subcutaneously into athymic nude mice, and volumes of the resulting tumors were then measured each week. Tumor volume in the Cep70 siRNA group was significantly
A

D

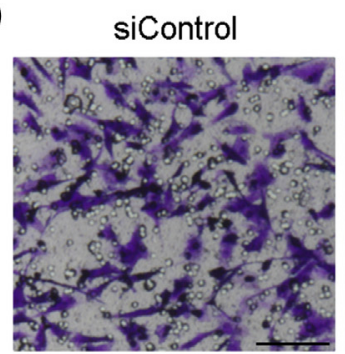

B

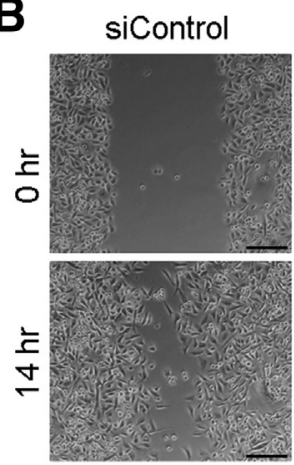

siCep70\#1

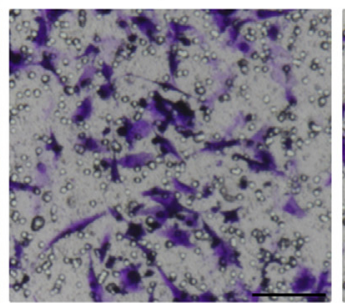

siCep70\#1

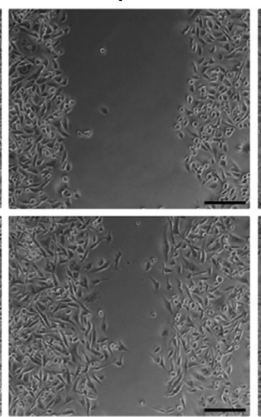

siCep70\#2

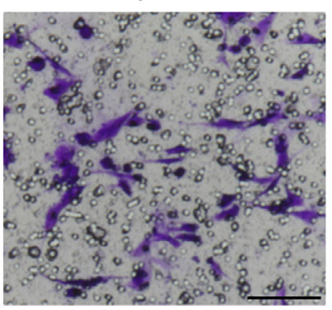

C

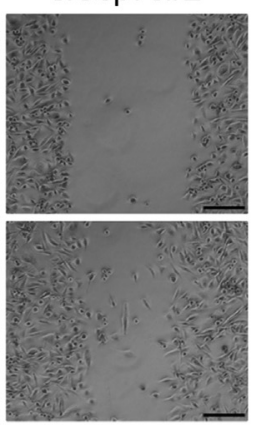

$\square$ siControl 口siCep70\#1 - siCep70\#2

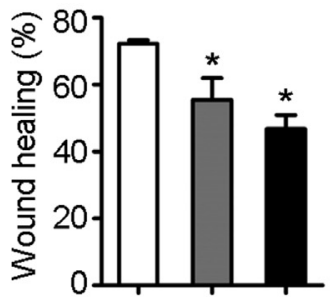

Figure 5 Effects of Cep70 on breast cancer cell migration and invasion in vitro. A: Immunoblot analysis of Cep70 and $\beta$-actin expression in MDA-MB-231 cells transfected with control or Cep70 siRNAs. B: Monolayers formed from control or Cep70 siRNA-transfected MDA-MB-231 cells were scratched, and wound margins were imaged 0 and 14 hours later. C: Experiments were performed as described in B, and the extent of wound healing was quantified. D: Control or Cep70 siRNA-transfected MDA-MB-231 cells were plated on inserts precoated with Matrigel. Cells that migrated through the porous membrane were then stained with crystal violet. E: Experiments were performed as described in $\mathbf{D}$, and the number of migrated cells was counted. Data are expressed as means \pm SD. ${ }^{*} P<0.05$ versus control. Scale bars $=100 \mu \mathrm{m}$. Cep70, centrosomal protein 70; si, small interfering. 
decreased compared with that of the control group (Figure 4F). In addition, the mass of tumors isolated from the Cep70 siRNA group was reduced compared with the control group (Figure 4G). We sectioned tumors isolated from mice and performed immunohistochemical staining of Cep70. As shown in Supplemental Figure S2, A and B, the knockdown effect still persisted in the tumors. It is known that cancer is caused by an imbalance between apoptosis and cell proliferation; therefore, we further examined whether Cep70 affected apoptosis or cell proliferation in tumor growth. We sectioned tissues from the xenografts and performed TUNEL assay and found that the Cep70 siRNA-treated group exhibited higher apoptotic index than the control group (Supplemental Figure S2, C and D). In addition, the decreased expression level of Ki-67 showed that Cep70 knockdown inhibits cell proliferation (Supplemental Figure S2, E and F). Together, these results suggest that Cep70 contributes to the development of breast cancer.

\section{Effects of Cep70 on Breast Cancer Cell Migration and Invasion in Vitro}

To investigate a potential function for Cep70 in breast cancer cell motility, we conducted in vitro wound healing and Transwell migration assays. In vitro wound healing assays revealed that knockdown of Cep70 expression in
MDA-MB-231 cells significantly decreased the extent of wound closure (Figure 5, A-C). Moreover, Transwell migration assays showed that Cep70 depletion also significantly suppressed invasion of breast cancer cells (Figure 5, $\mathrm{D}$ and E). Together, these results indicate that Cep70 is important for the motility of breast cancer cells.

\section{Bioluminescence Imaging Analysis of the Effect of Cep70 on Breast Cancer Metastasis}

We then investigated whether Cep70 plays a role in breast cancer metastasis in mice. Mouse 4T1-luc cells transfected with control or Cep70 siRNAs were injected into the tail veins of $\mathrm{BALB} / \mathrm{c}$ mice. Bioluminescence imaging in the presence of luciferin was then used to analyze the location of 4T1-luc cells in tumor-bearing animals after 8 days. We found that knockdown of Cep70 expression significantly inhibited metastasis of 4T1-luc cells to the lung (Figure 6, $\mathrm{A}-\mathrm{C})$. Analysis of the luminescence photon flux in isolated tumors confirmed that Cep70 played a critical role in cancer metastasis (Figure 6, D and E). We then conducted hematoxylin and eosin staining to examine lung sections from tumor-bearing mice and found that Cep70 depletion significantly decreased the area covered by metastases in the lung (Figure 6, F and G). Taken together, these data indicate that Cep70 increases breast cancer metastasis in vivo.
A

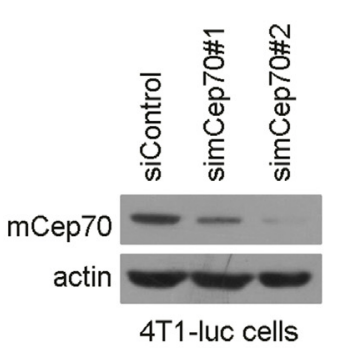

E

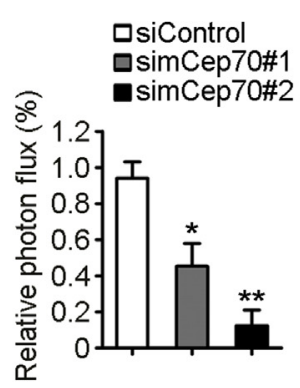

B

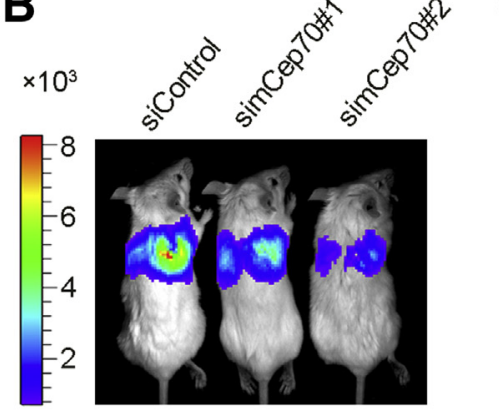

$\mathbf{F}$

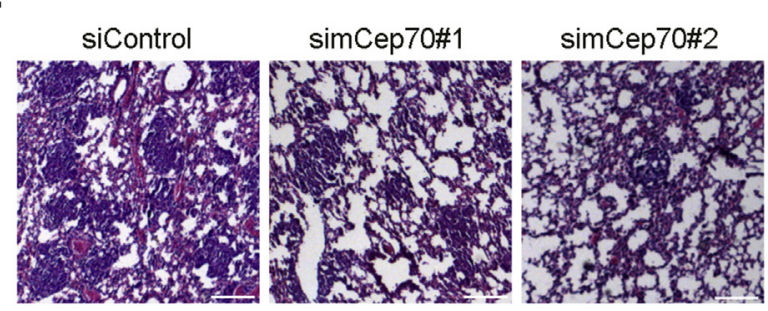

D
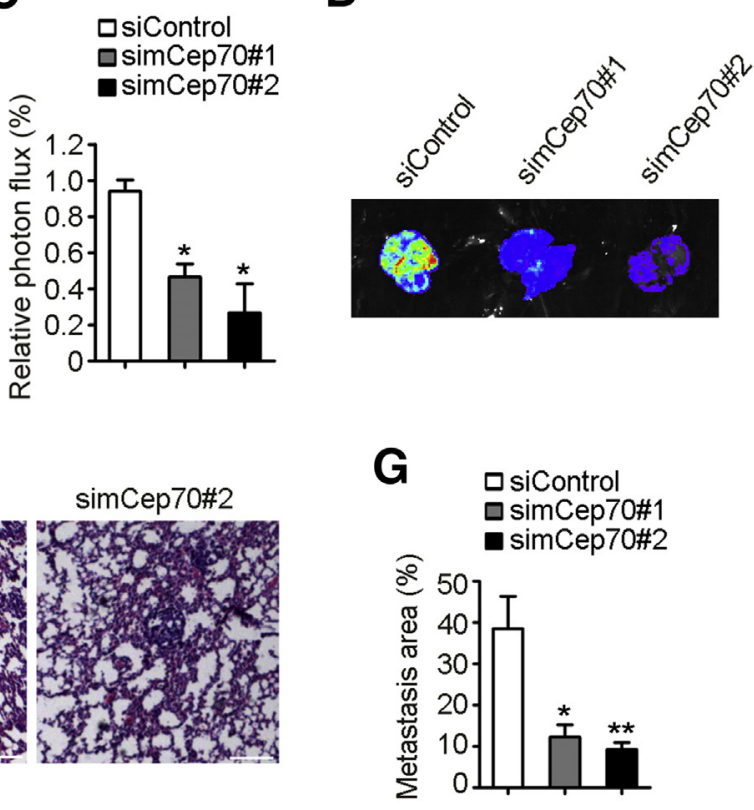

Figure 6 Bioluminescence imaging analysis of the effect of Cep70 on breast cancer metastasis. A: Immunoblot analysis of mCep70 and $\beta$-actin expression in 4T1-luc cells transfected with control or mCep70 siRNAs. B: 4T1-luc cells transfected with control or mCep70 siRNAs were injected into BALB/c mice. Mice were examined using a bioluminescence imaging instrument 8 days later. $\mathbf{C}$ : Experiments were performed as described in $\mathbf{B}$, and relative luminescence photon flux was quantified. D: Experiments were performed as described in B, and mice were sacrificed at the end point of day 8 . Tumors were isolated and analyzed by bioluminescence imaging. E: Examination of relative luminescence photon flux for tumors isolated as described in $\mathbf{D}$. F: Hematoxylin and eosin staining of lung sections from mice injected with control or mCep70 siRNA-transfected 4T1-luc cells. G: Experiments were performed as described in $\mathbf{F}$, and the percentage of area associated with metastasis was quantified. Data are expressed as means $\pm \mathrm{SD}$. ${ }^{*} P<0.05,{ }^{*} P<0.01$ versus control. Scale bars $=50 \mu \mathrm{m}$. Cep70, centrosomal protein 70; mCep70, mouse Cep70; si, small interfering. 


\section{Discussion}

Breast cancer results from a series of genetic alterations that can include inactivating mutations or deletions in tumor suppressor genes, activating mutations or amplification of oncogenes, and other types of abnormalities, such as epigenetic changes. ${ }^{23}$ Understanding the specific nature of these alterations and their impact on prognosis is important for determining the most beneficial treatment. The present study demonstrates that Cep70 is highly expressed in human breast cancer tissues and cell lines and that expression of Cep70 in breast cancer is correlated with clinicopathologic variables. These data suggest that Cep70 may have potential as a biomarker or target for the management of breast cancer, meriting further investigation.

Despite the observed elevation in Cep70 expression, its gene copy number was unchanged in breast cancer tissues or cell lines, indicating that Cep70 up-regulation is independent of gene amplification. Therefore, the precise genetic or epigenetic mechanism that leads to up-regulation of Cep70 expression remains elusive. In breast cancer, changes in DNA methylation and histone modifications, especially acetylation and methylation of histone lysines, are known to play essential roles in regulation of gene expression and nucleosome remodeling. ${ }^{24}$ For example, promoter methylation status modulates expression of p130 and BRCA1 in breast tumorigenesis. Thus, it is possible that epigenetic modifications, such as promoter demethylation and histone modifications, might contribute to Cep70 up-regulation in breast cancer.

Our results suggest that Cep70 enhances breast cancer metastasis by promoting the motility of breast cancer cells. This role points to a mechanism that involves Cep70mediated regulation of microtubules, because the dynamic properties of microtubules are essential for cell motility. Microtubule dynamics are tightly regulated by multiple factors that include microtubule-associated proteins, microtubule plus-end tracking proteins, and tubulin posttranslational modifications, such as detyrosination or acetylation. ${ }^{25}$ For example, the microtubule-associated protein Mdp3, the microtubule end-binding protein EB1, and the tubulin deacetylase HDAC6 all regulate cell motility through modulation of microtubule dynamics. ${ }^{22,26-29}$ Note that HDAC6 also contributes to breast cancer metastasis by increasing cell motility. ${ }^{30}$ Interestingly, our previous work has shown that Cep70 modulates microtubule dynamics through an interaction with HDAC6. ${ }^{31}$ It is possible that Cep70 overexpression contributes to breast cancer metastasis by modulating microtubule dynamics through its interaction with HDAC6 or by regulation of tubulin modification.

In addition to a potential role in control of microtubule dynamics, Cep70 could also contribute to the pathogenesis of breast cancer through changes in centrosome organization and/or function. Centrosome alterations may lead to abnormal microtubule nucleation and formation of disorganized mitotic spindles, contributing to chromosomal missegregation and aneuploidy. ${ }^{5,6}$ Our previous study has shown that Cep70 is necessary for the organization and orientation of the mitotic spindle. ${ }^{8}$ Therefore, it is conceivable that up-regulated expression of Cep70 deregulates the mitotic spindle, leading to genomic instability and tumorigenesis. It will be interesting to explore in further studies whether Cep70 is associated with abnormal centrosome architectures commonly observed in cancer cells.

\section{Acknowledgments}

We thank Shanxian Dongda Hospital for providing tissue samples and Dr. Guangming Gan for helping with immunohistochemistry.

\section{Supplemental Data}

Supplemental material for this article can be found at http://dx.doi.org/10.1016/j.ajpath.2016.11.005.

\section{References}

1. Siegel RL, Miller KD, Jemal A: Cancer statistics, 2016. CA Cancer J Clin 2016, 66:7-30

2. Anderson BO, Lipscomb J, Murillo RH, Thomas DB: Breast cancer. Edited by Gelband $\mathrm{H}$, Jha P, Sankaranarayanan R, Horton S. In Cancer: Disease Control Priorities. vol 3, ed 3. Washington, DC: World Bank, 2015

3. Braden AM, Stankowski RV, Engel JM, Onitilo AA: Breast cancer biomarkers: risk assessment, diagnosis, prognosis, prediction of treatment efficacy and toxicity, and recurrence. Curr Pharm Des 2014, 20: 4879-4898

4. Conduit PT, Wainman A, Raff JW: Centrosome function and assembly in animal cells. Nat Rev Mol Cell Biol 2015, 16:611-624

5. Godinho SA, Pellman D: Causes and consequences of centrosome abnormalities in cancer. Philos Trans R Soc Lond B Biol Sci 2014, 369:20130467

6. Rida PC, Cantuaria G, Reid MD, Kucuk O, Aneja R: How to be good at being bad: centrosome amplification and mitotic propensity drive intratumoral heterogeneity. Cancer Metastasis Rev 2015, 34:703-713

7. Pannu V, Mittal K, Cantuaria G, Reid MD, Li X, Donthamsetty S, McBride M, Klimov S, Osan R, Gupta MV, Rida PC, Aneja R: Rampant centrosome amplification underlies more aggressive disease course of triple negative breast cancers. Oncotarget 2015, 6: 10487-10497

8. Shi X, Sun X, Liu M, Li D, Aneja R, Zhou J: CEP70 protein interacts with gamma-tubulin to localize at the centrosome and is critical for mitotic spindle assembly. J Biol Chem 2011, 286:33401-33408

9. Shi X, Liu M, Li D, Wang J, Aneja R, Zhou J: Cep70 contributes to angiogenesis by modulating microtubule rearrangement and stimulating cell polarization and migration. Cell Cycle 2012, 11:1554-1563

10. Shi X, Wang J, Yang Y, Ren Y, Zhou J, Li D: Cep70 promotes microtubule assembly in vitro by increasing microtubule elongation. Acta Biochim Biophys Sin (Shanghai) 2012, 44:450-454

11. Wilkinson CJ, Carl M, Harris WA: Cep70 and Cep131 contribute to ciliogenesis in zebrafish embryos. BMC Cell Biol 2009, 10:17

12. Yang Y, Ran J, Liu M, Li D, Li Y, Shi X, Meng D, Pan J, Ou G, Aneja R, Sun SC, Zhou J: CYLD mediates ciliogenesis in multiple 
organs by deubiquitinating Cep70 and inactivating HDAC6. Cell Res 2014, 24:1342-1353

13. Shiratsuchi G, Kamiya R, Hirono M: Scaffolding function of the Chlamydomonas procentriole protein CRC70, a member of the conserved Cep70 family. J Cell Sci 2011, 124:2964-2975

14. Xie S, Qin J, Liu S, Zhang Y, Wang J, Shi X, Li D, Zhou J, Liu M: Cep70 overexpression stimulates pancreatic cancer by inducing centrosome abnormality and microtubule disorganization. Sci Rep 2016, 6:21263

15. Dong X, Liu F, Sun L, Liu M, Li D, Su D, Zhu Z, Dong JT, Fu L, Zhou J: Oncogenic function of microtubule end-binding protein 1 in breast cancer. J Pathol 2010, 220:361-369

16. Wang H, Liu B, Zhang C, Peng G, Liu M, Li D, Gu F, Chen Q, Dong JT, Fu L, Zhou J: Parkin regulates paclitaxel sensitivity in breast cancer via a microtubule-dependent mechanism. J Pathol 2009, 218:76-85

17. Sun X, Liu M, Hao J, Li D, Luo Y, Wang X, Yang Y, Li F, Shui W, Chen Q, Zhou J: Parkin deficiency contributes to pancreatic tumorigenesis by inducing spindle multipolarity and misorientation. Cell Cycle 2013, 12:1133-1141

18. Liu M, Wang X, Yang Y, Li D, Ren H, Zhu Q, Chen Q, Han S, Hao J, Zhou J: Ectopic expression of the microtubule-dependent motor protein Eg5 promotes pancreatic tumourigenesis. J Pathol 2010, 221:221-228

19. Sun L, Gao J, Dong X, Liu M, Li D, Shi X, Dong JT, Lu X, Liu C, Zhou J: EB1 promotes Aurora-B kinase activity through blocking its inactivation by protein phosphatase 2 A. Proc Natl Acad Sci U S A 2008, 105:7153-7158

20. Yang Y, Liu M, Li D, Ran J, Gao J, Suo S, Sun SC, Zhou J: CYLD regulates spindle orientation by stabilizing astral microtubules and promoting dishevelled-NuMA-dynein/dynactin complex formation. Proc Natl Acad Sci U S A 2014, 111:2158-2163

21. Gao J, Sun L, Huo L, Liu M, Li D, Zhou J: CYLD regulates angiogenesis by mediating vascular endothelial cell migration. Blood 2010, 115:4130-4137
22. Tala, Xie S, Sun X, Ran J, Zhang L, Li D, Liu M, Bao G, Zhou J: Microtubule-associated protein Mdp3 promotes breast cancer growth and metastasis. Theranostics 2014, 4:1052-1061

23. Byler S, Goldgar S, Heerboth S, Leary M, Housman G, Moulton K, Sarkar S: Genetic and epigenetic aspects of breast cancer progression and therapy. Anticancer Res 2014, 34:1071-1077

24. Basse C, Arock M: The increasing roles of epigenetics in breast cancer: implications for pathogenicity, biomarkers, prevention and treatment Int J Cancer 2015, 137:2785-2794

25. Etienne-Manneville S: From signaling pathways to microtubule dynamics: the key players. Curr Opin Cell Biol 2010, 22: 104-111

26. Sun X, Shi X, Liu M, Li D, Zhang L, Liu X, Zhou J: Mdp3 is a novel microtubule-binding protein that regulates microtubule assembly and stability. Cell Cycle 2011, 10:3929-3937

27. Hubbert C, Guardiola A, Shao R, Kawaguchi Y, Ito A, Nixon A, Yoshida M, Wang XF, Yao TP: HDAC6 is a microtubule-associated deacetylase. Nature 2002, 417:455-458

28. Li D, Sun X, Zhang L, Yan B, Xie S, Liu R, Liu M, Zhou J: Histone deacetylase 6 and cytoplasmic linker protein 170 function together to regulate the motility of pancreatic cancer cells. Protein Cell 2014, 5: 214-223

29. Li D, Gao J, Yang Y, Sun L, Suo S, Luo Y, Shui W, Zhou J, Liu M: CYLD coordinates with EB1 to regulate microtubule dynamics and cell migration. Cell Cycle 2014, 13:974-983

30. Saji S, Kawakami M, Hayashi S, Yoshida N, Hirose M, Horiguchi S, Itoh A, Funata N, Schreiber SL, Yoshida M, Toi M: Significance of HDAC6 regulation via estrogen signaling for cell motility and prognosis in estrogen receptor-positive breast cancer. Oncogene 2005, 24: $4531-4539$

31. Shi X, Yao Y, Wang Y, Zhang Y, Huang Q, Zhou J, Liu M, Li D: Cep70 regulates microtubule stability by interacting with HDAC6. FEBS Lett 2015, 589:1771-1777 